\title{
Upward Cocurrent Annular Flow of Air and Water in Smooth Tubes
}

\author{
SEYMOUR CALVERT AND BRYMER WILLIAMS \\ University of Michigan, Ann Arbor, Michigan
}

Two-phase fluid flow is distinguished from single-phase flow in two respects: (1) the cross section for flow of either fluid is not defined by the conduit alone and (2) not only the extent but the manner of frictional energy exchange for each fluid depends on the individual rates of flow for both fluids. It was believed therefore that an empirical approach would not adequately describe the various situations encountered in two-phase flow, and so study was undertaken to obtain some understanding of the mechanisms of the flow of liquid with a free su rface and the momentum exchange between fluids at that surface. It resulted in the development of a method of predicting liquid holdup and pressure drop for flowing systems in which the liquid, lifted by the gas flowing as a central core, moves upward as an annular film along the pipe wall.

In order to clarify the relationship of annular flow to the entire range of vertical two-phase flow modes, a discussion of vertical two-phase flow is presented, followed by an analysis of the special case of vertical, upward, annular flow; a description of the experimental work; and a comparison of experimental data with predictions.

The possible modes of vertical, two-phase flow are illustrated in the sketches in Figure 1, the conditions of liquid and gas flow under which the modes occur being indicated by the positions of the letters on the plot of pressure drop vs. air rate, shown as the bottom

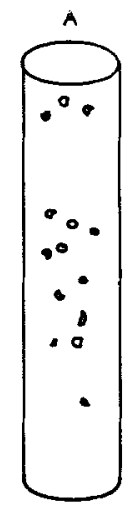

AERATED

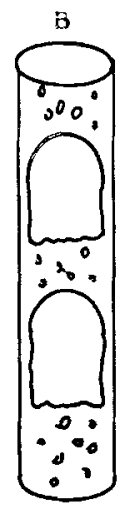

PISTON

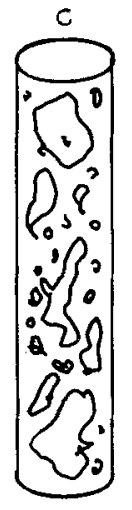

CHURN
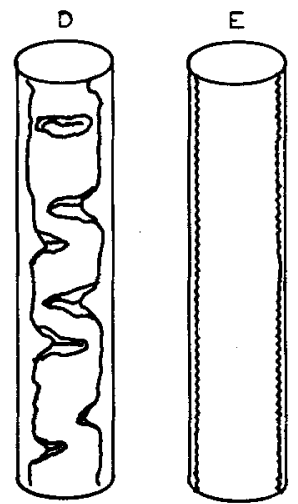

WAVE ANNULAR ENTRAINMENT

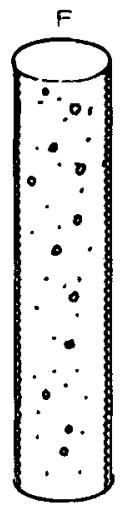

DROP ENTRAINMENT

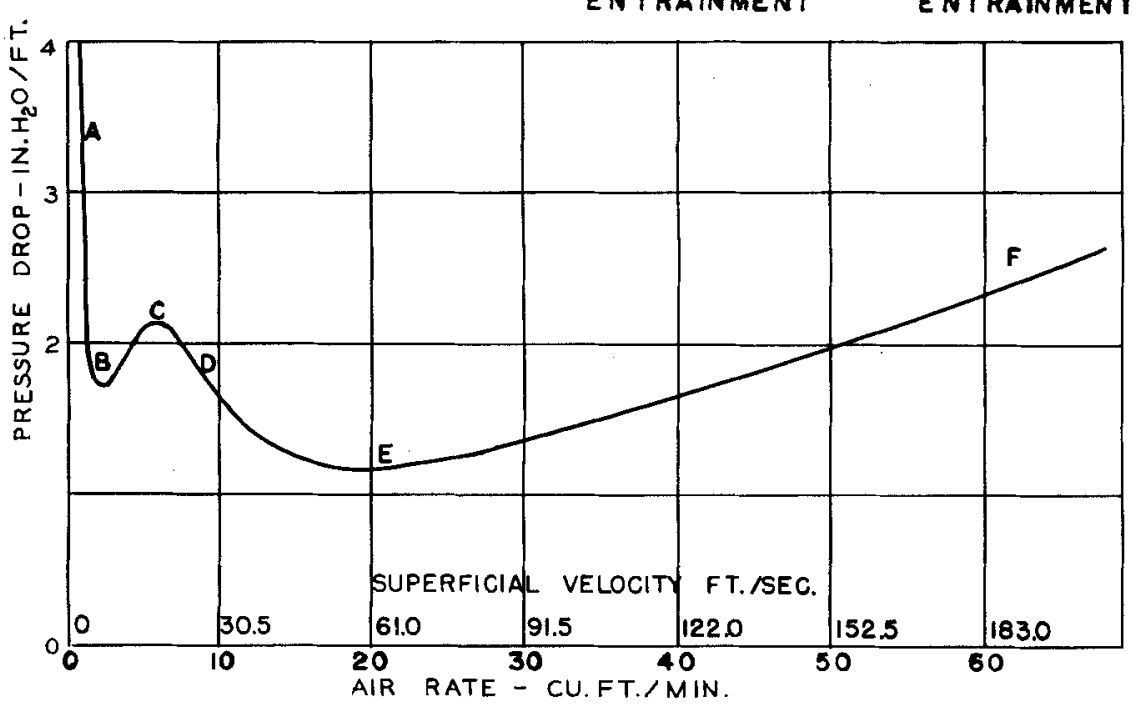

Frg. 1. Flow Patterns in Upward Two-phase Flow and a Plot of Pressure Drop vs. AIr Rate in a I-IN. I. D. Tube.

part of the figure. This pressure drop was drawn from experimental data (22) for the upward flow of air and $175 \mathrm{lb} . / \mathrm{hr}$. of water in a 1-in. I.D. plastic tube. At zero air rate the pressure drop is 12 in. of water/ft. of tube plus a small frictional loss. When a small amount of air is introduced continuously, it is dispersed and the pressure drop with increasing air rate approaches a minimum as the density of the mixture in the tube decreases. This is the mode of flow in ordinary gas lift. At about this minimum the flow mechanism changes from aerated, in which the air flows as small bubbles, to piston flow, in which the air flows as large, bullet-shaped bubbles. As the air rate is further increased, the pistons become unstable and the flow mechanism passes through the regimes of churn and of wave entrainment. These regimes may be thought of as merely the transition region between piston and annular flow since the violent, patternless agitation of churn flow gives way to the more placid, but still erratic, movement of waves superimposed on a film of water and finally to the uniform motion of annular flow. At some higher air rate drops of water will be pulled from the crests of the small waves on the annular film and entrained.

Thus annular flow is bounded by the regimes of wave and drop entrainment. The lower limit of annular flow is at the second minimum pressure drop (at about 18 cu.ft./min. in Figure 1). The upper limit has not been observed within the range of experimental data 
available (the position of point $F$ in Figure 1 is merely illustrative).

The regime of upward, cocurrent annular flow is defined as that in which the liquid flows as a uniform annular film on the pipe wall while the gas flows as a central core inside the liquid annulus. Two simultaneous processes take place in annular flow: the liquid film flows as a result of the drag exerted on it by the gas and the flowing gas loses a quantity of energy which is dictated by the amount of liquid flowing. A mathematical description of the flow of the liquid film is derived here through an analytical procedure based on the Prandtl and Von Karman(17) theories of turbulent flow, and the result is a general expression which is valid for any liquid film. This special derivation is necessitated by the

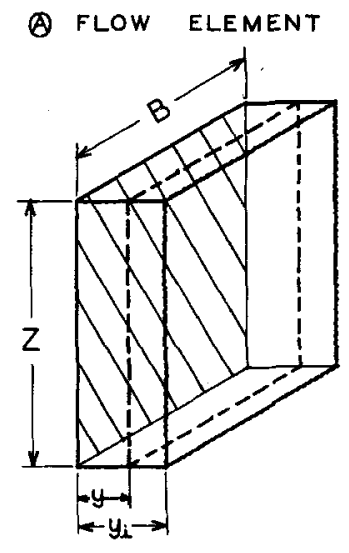

C) SHEAR DISTRIBUTION

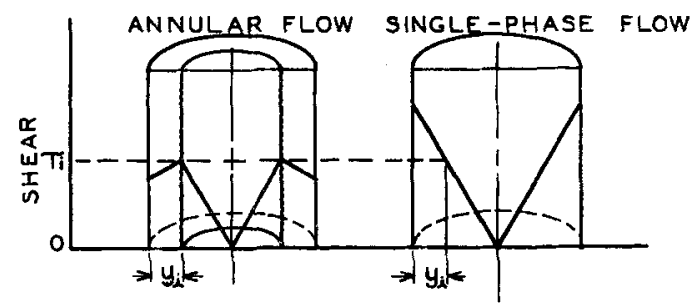

Fig 2. Flow Element and Illustrations of Force and Shear Distributions.

significant effect of gravity on the vertical flow of the liquid and by special consideration which must be given to the effect of the solid boundary, which is of more importance in film flow than in a fullrunning pipe.

Gas loses momentum to the liquid in annular flow in what may be considered two separate mechanisms. One is the loss by "skin friction," where the gas directly in contact with the boundary is moving slowly and the retarding effect
(3) FORCE BALANCE

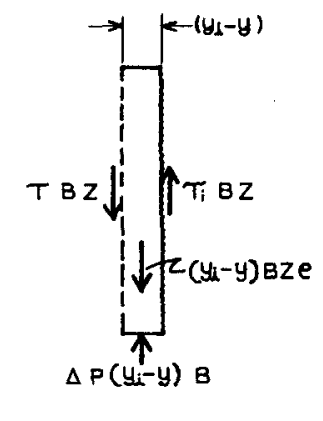

is exerted gradually into the main stream. The other mechanism is that of "profile drag," where gas of high velocity impinges on a projecting object and loses a part of its kinetic energy. The obstacles in the path of the gas are the liquid waves. Their projected areas determine the drag area and their shapes and spacing determine the drag coefficient, which represents the fraction of the kinetic energy of impingement lost.

Skin friction can be determined in the usual manner of predicting pressure drop for single-phase flow through smooth pipes by employing the friction factor. The determination of profile drag requires a knowledge of the velocity of the gas striking the projections, the area of the projections, and the drag coefficient. The combined ef-

$$
\begin{aligned}
\tau(B Z)= & \tau_{i}(B Z)+\Delta P\left(y_{i}-y\right) B- \\
& \left(y_{i}-y\right) B Z \rho_{\perp}
\end{aligned}
$$

where

$\tau=$ shear (force per unit area $B Z$ ) at any position $y$ in the film, lb./sq.ft.

$\tau_{i}=$ shear at the air-water interface, lb./sq.ft.

$y=$ distance from the solid boundary, ft.

$y_{i}=$ film thickness, ft.

$B=$ width of the film, $\mathrm{ft}$.

$Z=$ height of the film

$\triangle P=$ pressure drop in the direction of $Z$, lb./sq.ft.

$e_{L}=$ density of the liquid, lb./cu.ft.

Figure $2 a$ is a sketch of an elemental section of the annular flow system, and Figure $2 b$ illustrates the force balance over a slice of this element. It should be noted that the model element is simplified by being considered a flat plane rather than a cylinder. Figure $2 c$ represents possible shear distributions in both vertical, upward, annular flow and single-phase flow in tubes. The shear distribution in both cases is given by a force balance; that for single-phase vertical flow is

$$
\tau=\Delta P \frac{\pi r^{2}}{2 \pi r Z}-\frac{\pi r^{2} Z \rho}{2 \pi r Z}
$$

fect of these quantities on profile
drag had to be determined experimentally.

Derivations of equations describing the liquid film and the determination of profile drag from experimental data will be given in the following pages. The rate of the liquid film can be defined in terms of the pressure drop and the thickness of the film. The pressure drop is also related to the air velocity and the liquid-film thickness. A combination of these two relation- or

$$
\tau=\frac{r}{2}\left(\frac{\Delta P}{Z}-\rho\right)
$$

where $r$ is the radius.

Equation (1) has been derived for annular film flow, and as $\tau_{i}$ can be related to $\frac{\triangle P}{Z}$ by Equation (2), 
the shear distribution for a film can be written as

$$
\begin{gathered}
\tau=\left(\frac{\Delta P}{Z}-\rho_{\mathrm{G}}\right)\left(\frac{r_{o}-y_{i}}{2}\right)+ \\
\left(\begin{array}{c}
\left.y_{i}-y\right)\left(\frac{\Delta P}{Z}-\rho_{\mathrm{L}}\right.
\end{array}\right)
\end{gathered}
$$

To clarify the disconcerting experimental observation that a water film can flow up a tube with a pressure drop of much less than $1 \mathrm{ft}$. of water/ft. of height (see Figure 1), one may compare the shear distributions for single- and two-phase flow.

If the fluid in the single-phase case has the same density as that flowing in the film, and the shear at a distance $y_{i}$ from the wall is the same in both cases, as shown in Figure $2 c$, the pressure drops in the two cases are related by Equation (5):

$$
\frac{\Delta P_{\mathrm{A}}}{Z}-\rho_{\mathrm{G}}=\frac{\Delta P_{\mathrm{s}}}{Z}-\rho_{\mathrm{L}}
$$

where

$$
\Delta P_{A}=\text { pressure drop for annular }
$$
flow, lb./sq.ft.

$\Delta P_{s}=$ pressure drop for singlephase flow, lb./sq.ft.

$P_{G}=$ density of gas phase, lb./cu.ft.

If the two fluids in question are air and water at atmospheric pressure, $\rho_{a}$ is negligible. The pressure drop required to produce a given $\tau_{i}$ is lower for annular flow than for single-phase flow. It is seen that the shear distribution within the annular film is not the same as it would be for the same film in single-phase flow of the same fluid; therefore equations which describe single-phase flow will not describe vertical annular-film flow. The two cases will approach each other when the pressure drop is so high that it overshadows the effect of liquid density upon the shear distribution.

With the shear distribution defined, the next problem is to describe the manner in which the fluid will act to implement this distribution of forces. One element of a fluid body can exert a force on an adjacent element only if there is a difference in velocity between them. Consequently the ultimate goal of this next analysis is the determination of a velocity pattern that will sustain the annular-flow shear distribution.

One can predict whether the flow pattern will be laminar or turbulent in single-phase flow by the use of the Reynolds criterion of turbulence. Unfortunately this criterion becomes meaningless when it is applied to two-phase flow, because of the absence of a term which considers the phase-to-phase energy transfer. A more general criterion which has been developed here and will be discussed in a later section indicates that turbulent flow is the predominant type encountered in vertical, annular water-film flow. All further attention is, then, given to the turbulent flow of liquid films.

\section{TURBULENT-FLOW CONCEPTS: ANNULAR FLOW}

The application of the Prandtl theory to single-phase flow has been well discussed (1). Several simplifying assumptions which are made in that treatment, however, are not sufficiently accurate to justify its adoption for liquid-film flow. The fact that the bulk of the fluid in single-phase flow moves in the central turbulent core masks errors in the consideration of the fluid close to the boundary. In annular flow the liquid is so greatly influenced by the solid boundary that one must be especially concerned with fluid behavior in this region and the assumptions must be evaluated for this special case.

First, it may be assumed that the motion of the fluid at any point is dependent upon the local shear stress. With this concept Von Karman (1) arrived at a definition of turbulent flow which is almost identical with Prandtl's. Both definitions are valid for single-phase flow, as is shown by their use in correlating experimentally determined velocity profiles.

Next there are assumptions which are implied by the first condition. If the local velocity gradient is dependent on the local shear alone, then the mixing-length distribution in a film of liquid must be the same as that in a pipe running full of Iiquid. Likewise the thickness, $\delta$ of the laminar layer must depend on the shear at the laminar layer.

The first of these implications is quite sound as the mixing length is a function of distance from the wall and not of the flow conditions. The second assumption is more difficult to justify as the point conditions which determine thickness of the laminar layer $\delta$ are not known. The only definite information available is the empirical relationship giving $\delta$ as a function of kinematic viscosity and shear at the wall for single-phase flow.

$$
\frac{\delta}{D}=\frac{N}{\operatorname{Re}} \sqrt{\frac{8}{f}}=N \frac{\mu}{\rho \sqrt{\frac{\tau_{o} g}{\rho}}}
$$

where

$\delta=$ thickness of the laminar layer

$D=$ pipe diameter.

$N=$ a constant for all fluids

$R e=$ Reynolds number

$f=$ friction factor

$\tau_{0}=$ shear at pipe wall

$\mu=$ absolute viscosity

This relationship must be modified and utilized to define $\delta$ for annular flow although it is not yet directly applicable. It was shown earlier (see Figure 2) that while the shear at the wall defines a unique shear distribution for single-phase flow, it does not for annular flow. Consequently, it is assumed that the shear stress at $\delta$ in an annular film bears the same relationship to $\delta$ that wall shear does in single-phase flow.

\section{DERIVATION OF FILM-FLOW EQUATIONS}

The mathematical description of film flow is attained in two steps. First the differential equations expressing velocity gradient are integrated to give a description of velocity distribution. Once the velocity distribution is known, the rate of flow can be determined by another integration process. This is done first for the turbulent layer and next for the laminar layer.

The shear is related to the velocity gradient which it produces by

$$
\tau=\frac{\rho}{g} l^{2}\left(\frac{d u}{d y}\right)^{2}
$$

where $l$ is the Prandtl mixing length and $u$ is the velocity in the axial direction at a distance $y$ from the wall.

The shear $\tau$ may be eliminated by combining Equations (4) and (7):

$$
\begin{gathered}
\frac{\rho_{\mathrm{L}}}{g} l^{2}\left(\frac{d u}{d y}\right)^{2}=\left(\frac{\Delta P}{Z}-\rho_{\mathrm{G}}\right) \\
\left(\frac{r_{o}-y_{i}}{2}\right)+\left(\frac{\Delta P}{Z}-\rho_{\mathrm{L}}\right)\left(y_{i}-y\right)
\end{gathered}
$$

The velocity distribution is found by integration of Equation (8), which states the relationship between local velocity gradient and local shear. To do this it is assumed that $\left(r_{o}-y_{i}\right) \approx r_{o}$ and that $\left(y_{l}-y\right) \frac{\Delta P}{Z}$ is negligible. This 
amounts to taking an average value for the shear due to $\frac{\Delta P}{\eta}$ :

$$
\begin{gathered}
\frac{r_{o}}{2}-\left(\frac{\Delta P}{Z}\right)-\left(y_{i}-y\right) \rho= \\
\frac{\rho}{g} l^{2}\left(\frac{d u}{d y}\right)^{2}
\end{gathered}
$$

The mixing length $l=0.4 y$, as evaluated by Nikuradse (1).

$$
\begin{gathered}
\frac{r_{o}}{2}\left(\frac{\Delta P}{Z}\right)-\left(y_{i}-y\right) \rho= \\
\frac{\rho}{g}(0.4 y)^{2}\left(\frac{d u}{d y}\right)^{2}
\end{gathered}
$$

Next, if the equation is rearranged and the square root taken $\frac{d u}{d y}=\frac{\sqrt{g}}{\left[\frac{r_{o}}{2 \rho} \frac{\Delta P}{Z}+\left(y_{i}-y\right)\right]^{\frac{1}{2}}}$; let

$$
A=\frac{r_{o}}{2 \rho} \frac{\Delta P}{Z}
$$

To obtain a simple form after this equation is integrated, the term in the brackets is expanded according to the binomial theorem:

$$
\begin{gathered}
\frac{d u}{d y}=\frac{\sqrt{A g}}{0.4 y}+\frac{\sqrt{g}}{2(0.4 y) \sqrt{A}} \frac{\left(y-y_{i}\right)}{\sqrt{A}} \\
-\frac{\sqrt{g}\left(y-y_{i}\right)^{2}}{8(0.4 y) A^{3 / 2}}+\cdots
\end{gathered}
$$

with the condition for convergence that

$$
\left[\frac{\left(y-y_{i}\right)}{\frac{r_{o}}{2 \rho} \frac{\Delta P}{Z}}\right]^{2} \leqq 1
$$

The significance of this condition may be more readily seen after rearrangement of (17) to give

$$
\left|\left(y-y_{i}\right) \rho\right| \leqq\left|\frac{r_{o}}{2}-\frac{\Delta P}{Z}\right| \leqq\left|\tau_{i}\right|
$$

This means that the series will not converge for the values of $\tau_{0}=0$ which would occur if the gravity terms were equal to the interfacial shear. The necessary condition is obtained in vertical, upward, annular flow, and so the series is convergent for the case.

Equation (12) may be integrated to give

$$
u=\frac{\sqrt{A g}}{0.4} \ln y+\frac{\sqrt{g}}{2(0.4) \sqrt{A}}
$$

$$
\begin{gathered}
\left(y-y_{i} \ln y\right)-\frac{\sqrt{g}}{2(0.4) A^{3 / 2}} \\
\left(\frac{y_{2}}{2}+2 y y_{i}+y_{i} \ln y\right)+\ldots+C(15)^{*}
\end{gathered}
$$

The velocity distribution in the turbulent region is thus given by

$$
\begin{aligned}
& {[u]_{\delta}^{y}=\left[\left(\frac{\sqrt{\frac{r_{o}}{2_{\rho}}-\frac{\Delta P}{Z}} g}{0.4}\right) \ln y+\right.} \\
& \left.\frac{\sqrt{g}}{0.8 \sqrt{\frac{r_{o}}{2 \delta} \frac{\Delta P}{Z}}}\left(y-y_{i} \ln y\right)\right]_{\delta}^{y}
\end{aligned}
$$

To simplify, if

$$
\begin{aligned}
& \frac{\sqrt{\frac{r_{o}}{2 \delta} \frac{\overline{\Delta P}}{Z} g}}{0.4}=X \\
& \frac{\sqrt{g}}{0.8 \sqrt{\frac{r_{o}}{2 \delta}} \frac{\Delta P}{Z}}=Y
\end{aligned}
$$

then

$$
\begin{aligned}
& u-u_{\delta}=X \ln \frac{y}{8}+Y \\
& {\left[\left(y-y_{i} \ln y\right)-\delta+y_{i} \ln \delta\right]}
\end{aligned}
$$

Equation (17) describes the velocity distribution in the turbulent region in terms of a base velocity, $u \delta$, which can be determined by application of laminarflow equations in the region from the wall to $\delta$. The quantity of liquid flowing through the film can be computed by integrating an equation giving the product of velocity and the differential area for flow; that is,

* It is interesting to note that this is in the form of the Prandtl velocity distribution for ull pipes where

$$
u=\frac{u^{*}}{k} \ln y+C
$$

and in the same symbols, for Equation (15),

$$
\begin{gathered}
u=\frac{u^{*}}{k} \ln y+\frac{g}{2 k u^{*}} \\
\left(y-y_{i} \ln y\right)+\ldots+C
\end{gathered}
$$

where

$$
u^{*}=\frac{\sqrt{r_{o}} \bar{g}}{\rho} \text { and } k=\frac{l}{y}=0.4
$$

In Equation $(15 \alpha)$ the terms after the first on the right side account for the gravity effect. Without these terms Equation (15a) would be identical with Prandtl's. The first two terms on the right side of (15) are sufficient to represent the series with reasonable accuracy, and accordingly the remaining terms are neglected.

$$
L=2 \pi r \int_{0}^{y^{i}} u d y
$$

The volume rate of flow, $L$, is defined as

$$
\begin{gathered}
L=2 \pi r_{o}\left[\int_{o}^{\delta} u d y+\int_{\delta}^{y_{i}} u_{\delta} d y+\right. \\
\left.\int_{\delta}^{y_{i}}\left(u-u_{\delta}\right) d y\right]
\end{gathered}
$$

The first two terms in the brackets in Equation (19) depend on the laminar-flow mechanism and $\delta$ so they are called the laminar terms. As the third term involves the turbulent-flow mechanism, it is called the turbulent term.

The laminar terms may be approximated as

$$
L_{l a m}=2 \pi r_{o}\left[u_{\delta}\left(y_{i}-\frac{\delta}{2}\right)\right]
$$

In the derivation of the foregoing equation it was assumed that the laminar velocity gradient may be considered constant for the calculation of $L_{\text {lam }}$ although it cannot be so assumed for the calculation of $u$

The volume rate of flow in the turbulent term is

$$
\begin{gathered}
L_{t}=2 \pi r_{o} \int_{\delta}^{y^{i}}\left(u-u_{\delta}\right) d y=2 \pi r_{o} \\
\int_{\delta}^{y^{i}}\left[X \ln \frac{y}{\delta}+Y\left(y-\delta-y_{i}\right)\right. \\
\left.\ln \frac{y}{\delta}\right] \ln y
\end{gathered}
$$

Integration of Equation (21) gives Equation (22):

$$
\begin{gathered}
\frac{L_{t}}{2 \pi r_{o}}=\ln \frac{y_{i}}{\delta}\left(X y_{i}-Y y_{i}^{2}\right)- \\
\left(1-\frac{\delta}{y_{i}}\right)\left(X y_{i}\right)+\left(Y y_{i}^{2}\right) \\
\left(1-\frac{\delta}{y_{i}}\right)\left(\frac{3-\delta / y_{i}}{2}\right)
\end{gathered}
$$

and since $\delta / y_{i} \ll 3$

$$
\begin{aligned}
& \frac{L_{t}}{2 \pi r_{o}}=\ln \frac{y_{i}}{\delta}\left(X y_{i}-Y y_{i}^{2}\right)- \\
& \left(1-\frac{\delta}{y_{i}}\right)\left(X y_{i}-\frac{3}{2} Y y_{i}^{2}\right)
\end{aligned}
$$




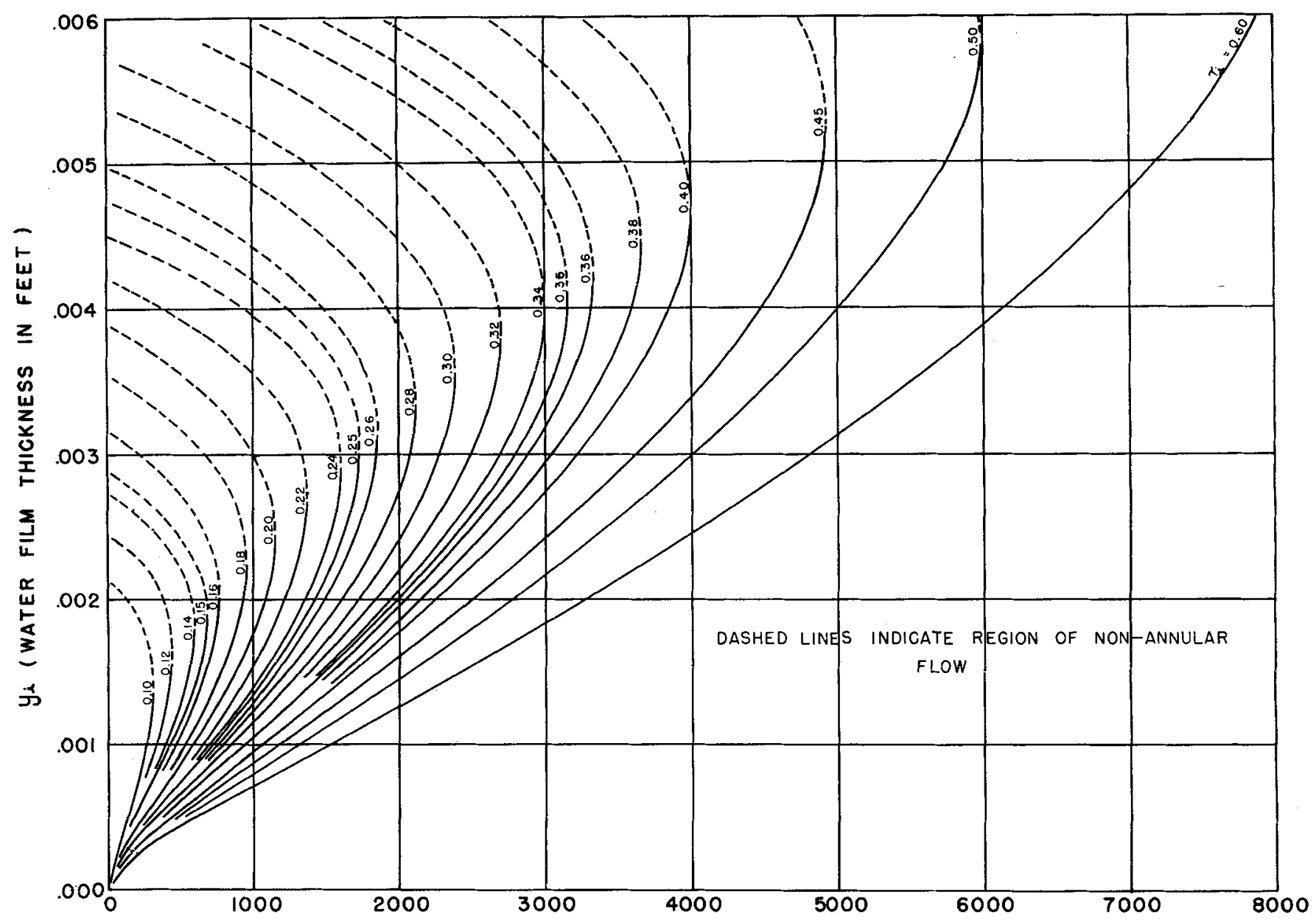

$L$ (POUNDS OF WATER / HOUR / FOOT OF CIRCUMFERENGE)

Fig. 3. Predicted Film Thickness vs. Water Rate with Interfacial Shear as the Parameter.

The final form of the volume rate of flow equation is the sum of $L_{l a m}$ and $L_{t}$ as given below:

$$
\begin{gathered}
\frac{L}{2 \pi r_{o}}=\left[u\left(y_{i}-\frac{\delta}{2}\right)+\right. \\
\ln \frac{y_{i}}{\delta}\left(X y_{i}-Y y_{i}^{2}\right)- \\
\left.\left(1-\frac{\delta}{y_{i}}\right)\left(X y_{i}-\frac{3}{2} Y y_{i}^{2}\right)\right]
\end{gathered}
$$

The remaining task is to evaluate $u \delta$ and $\delta$ in terms of $\frac{\Delta P}{Z}$ and $y_{i}$. If $\delta$ is determined by $\tau_{\delta}$, one can evaluate $\delta$ by solving Equations (25) and (26) below:

$$
\delta=\frac{N \mu}{\rho u_{\delta}}=11.6 \frac{\mu}{\rho \sqrt{\frac{\tau_{\delta} g}{\rho}}}
$$

and

$$
\tau_{\delta}=\tau_{i}-\left(y_{i}-\delta\right) \rho
$$

The value of 11.6 has been taken for the value of the constant. $N$; this is an assumption since there is some dispute as to the exact value of $N$ although 11.6 appears to be good average (1).

Next $u_{\delta}$ can be determined by solving Equation (27), which is the definition of viscosity

$$
\begin{gathered}
\int_{o}^{u_{\delta}} d u=-\frac{g}{\mu} \\
\int_{0}^{\delta}\left[\tau_{i}-\rho\left(y_{i}-y\right)\right] d y
\end{gathered}
$$

which gives upon integration

$u_{\delta}=\frac{g \rho}{\mu}\left[\left(\frac{\tau_{i}}{\rho}-y_{i}\right) \delta+\frac{\delta^{2}}{2}\right]$

\section{SUMMARY OF LIQUID-FILM FLOW MEASUREMENTS}

To summarize the preceding development, one may set up a procedure for determining the volume rate of flow, $L$, if $y_{i}, \omega, \%, \gamma_{0}$ and $\tau_{0}$ are known. The following stages are involved:

1. Determination of $\delta$ with Equations (25) and (26).

2. Determination of $\mu_{\delta}$ with Equation (28).

3. Determination of $L_{\text {lam }}$ with Equation (20).

4. Determination of $L_{t}$ with Equation (23).

5. Addition of $L_{l a m}$ and $L_{t}$ to give $L$.

The results of a series of computations under the foregoing procedure can be tabulated graphically in a plot of $y_{i}$ vs. $L$ with $\tau_{i}$ as a parameter. Figure 3 is such a plot for the upward flow of water films. The lines of constant shear are dotted in the region in which annular flow does not exist.

\section{THE CRITERION OF TURBULENCE}

The Reynolds criterion has been applied to two-phase flow by various workers who used a hydraulic diameter as the significant length. 
The success of such an approach is limited to special cases in which the interphase momentum exchange is either negligible or constant.

An example of this approach is the work of Friedman and Miller $(\gamma)$ on falling annular films in vertical pipes. They indicate that liquid films running downward while a stationary core of air fills the center of the pipe become turbulent at a Reynolds number of 1,000. Fallah, Hunter, and Nash (6) show that when another fluid, denser than air but lighter than water, is employed as the central core the critical Reynolds number is much less than 1,000 , even as low as 15 . Obviously, the flow rate of the film, its fluid properties, and the pipe diameter alone are not sufficient to define its state of motion.

In order completely to define the flow of each fluid in two-phase flow it is necessary to include the effect of momentum transfer between phases in the criterion of turbulence. One familiar quantity has this property although it is deficient in a significant length term: the thickness of the laminar layer $\delta$ set by the kinematic viscosity of and the shear in the fluid. The ratio of o to pipe diameter for singlephase flow is a function of the Reynolds number and the friction factor only(1). Since the friction factor becomes a function of Reynolds number alone rather than of Reynolds number and relative roughness as the Reynolds number decreases to values of about 2,100 , the ratio of $\delta$ to diameter has a single critical value corresponding to $R e=2,100$.

$\mathrm{By}$ analogy, the ratio of $\delta / y_{i}$ is taken as the criterion of turbulence for film flow. Film thickness replaces pipe diameter as the significant length in the ratio. A critical value of this ratio, determined from the data presented by Friedman and Miller by evaluating $\delta / y_{i}$ at $R e=1,000$, is

$$
\left(\frac{\delta}{y_{i}}\right)_{c}=\frac{11.6}{2.85 \sqrt{2.85 g}}=0.425
$$

This critical ratio marks the point of abrupt change from turbulent to laminar flow. If it is larger than 0.425 the flow pattern will be laminar. It is interesting to note that this criterion indicates that the film will become entirely laminar in character at a point where roughly half its thickness would flow by the laminar mechanism even if the flow were turbulent.

TABLE 1.-RANGE OF EXPERIMENTAL INVESTIGATION

Tube diameters, in . . . . . . . .

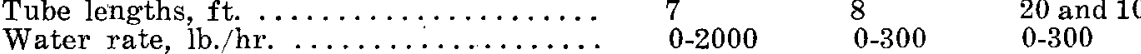

Air. rate, cu. ft./min. at test conditions .. $55-112 \quad 10-74 \quad 10-74$

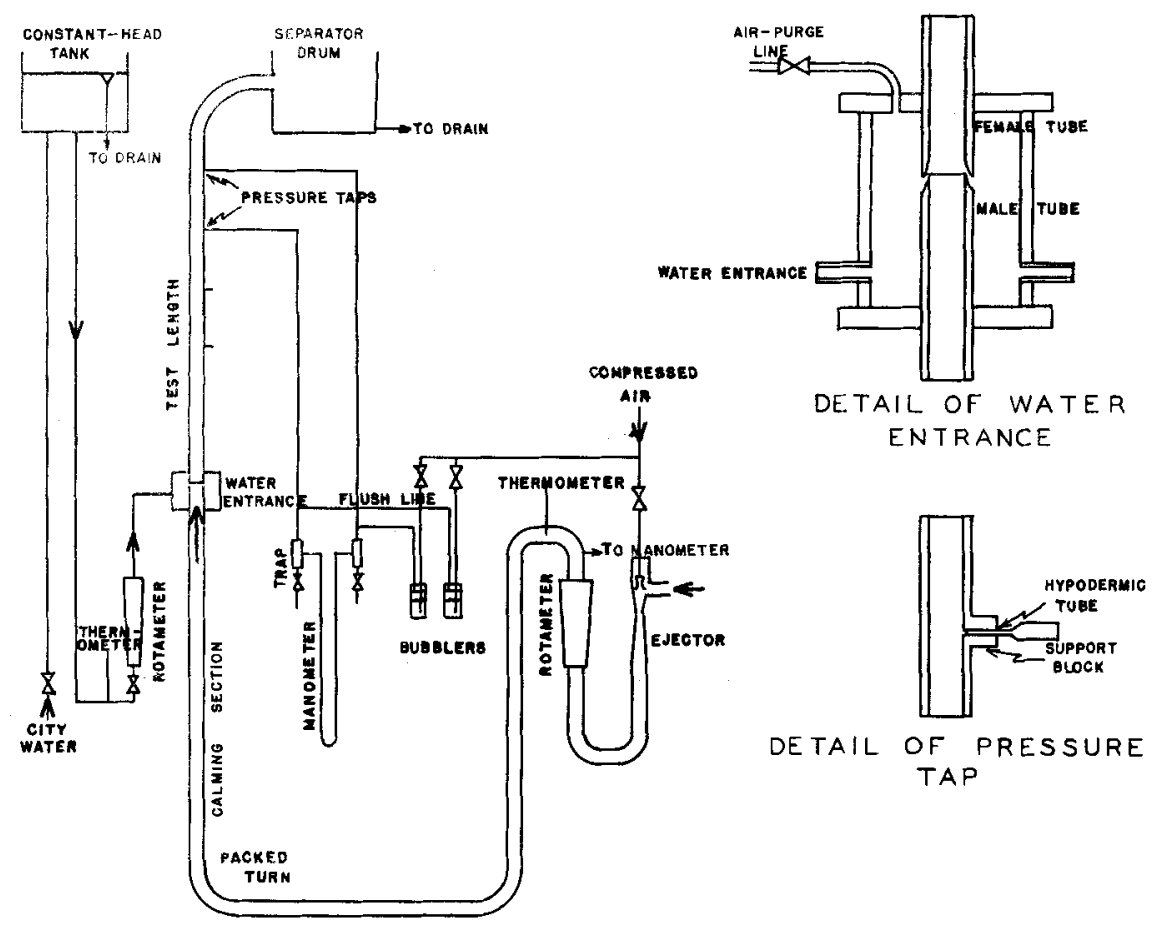

Fig. 4. Diagrams of Experimental Apparatus.

This criterion was applied to the case of vertical upward annular flow of water, and the indication was that the flow is turbulent throughout the regime.

\section{EXPERIMENTAL}

Theoretical considerations of the modes of flow predict the momentum transfer and pressure drop between the phases. It was necessary to obtain experimental data to establish the validity of these equations which define film flow and to evaluate certain coefficients. Data of B. H. Radford (18) for the upward flow of air and water in a 1-in. tube provided water-holdup information which was used to estimate liquid-film thickness. The present investigation provided pressure-drop data for the various tubes and flow conditions given in Table 1.

The system is shown in Figure 4. The equipment provides for metered air to enter the test pipe from a straightening section and metered water to enter through a circumferential slot in the bottom of the test section. Pressure drops were measured over 2 - or 3-ft. intervals along the tube with manometers connected with air-flushed pressure taps. This arrangement provided for measurement of entrance and exit effects, if any. Various entrance devices were tried, and as the annular ring shown in Figure 4 proved to be the most. stable, results reported are for its use. Typical data are shown in Figure 5 .

\section{EQUATION FOR PROFILE DRAG}

One can describe the profiledrag process mathematically by writing first that the shear produced is equal to a fraction of the stagnation pressure of the gas stream times the area of stagnation, as

$$
F=C_{\mathrm{D}}\left(\Delta P_{\mathrm{S}} A_{\mathrm{D}}\right) Z 2 \pi r_{o}
$$

where

$F=$ drag force, $1 b$.

$Z=$ length of pipe, ft.

$C_{D}=$ drag coefficient

$\Delta P_{s}=$ stagnation pressure, $1 \mathrm{~b} . / \mathrm{sq} . \mathrm{ft}$.

$A_{D}=$ projected area for drag, sq.ft. /sq.ft. inside pipe area.

$r_{o}=$ pipe radius, $\mathrm{ft}$.

The stagnation pressure is obtained from Bernoulli's equation as

$$
\frac{u_{\mathrm{A}}^{2}}{2 g}=\frac{\Delta P_{s}}{\rho}
$$

Thus

$$
F=C_{\mathrm{D}}\left(\frac{u_{\mathrm{A}}^{2} \rho_{\mathrm{G}} A}{2 g}\right) Z 2 \pi r_{\mathrm{D}}
$$



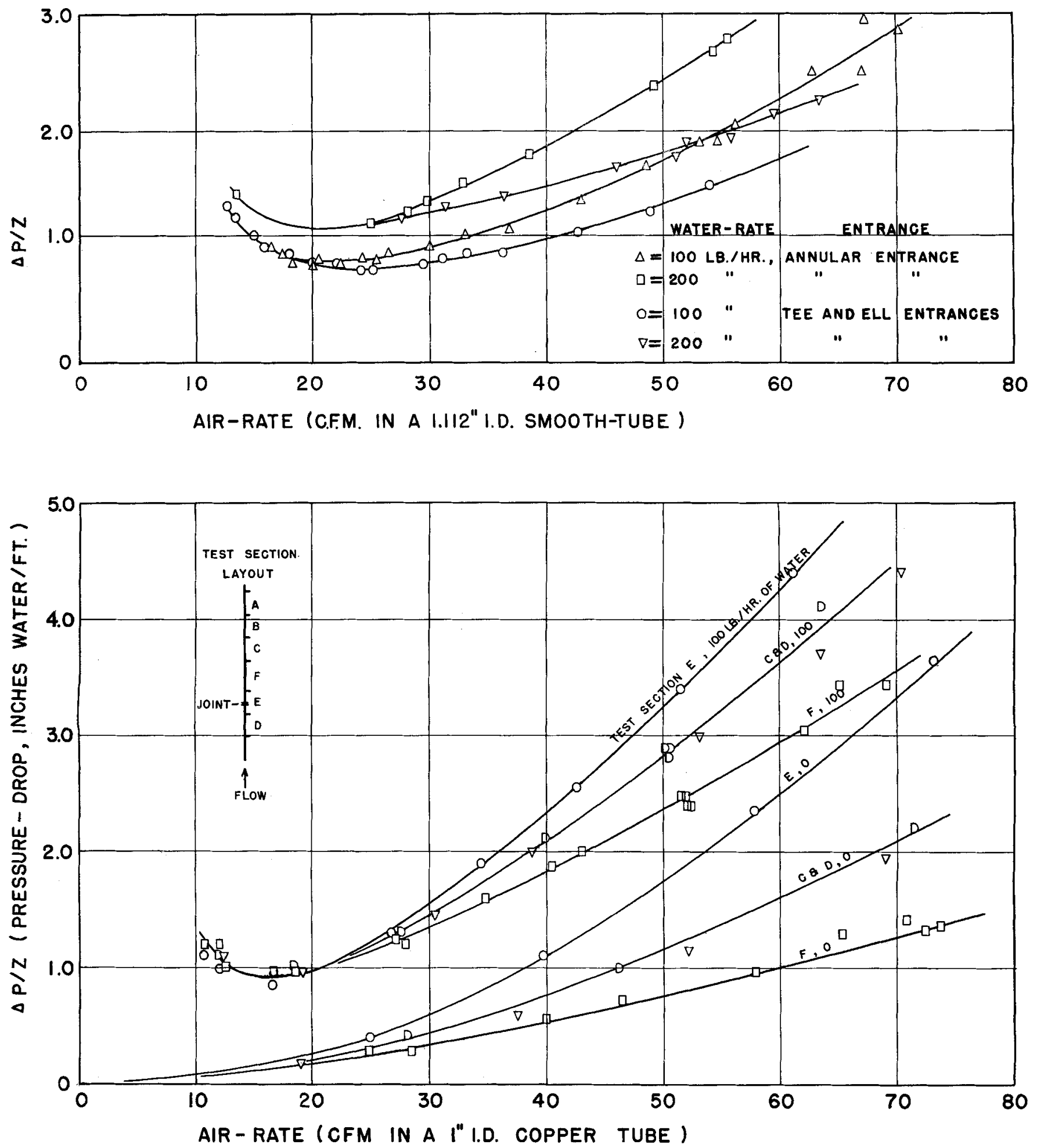

Fig. 5. Pressure-drop vs. Air-rate Plots for Various Water Entrances and for tube Roughness.

and also

$\Delta P \mathrm{E}=C_{\mathrm{D}}\left(\frac{u_{\mathrm{A}}^{2} \rho_{\mathrm{G}} A_{\mathrm{D}}}{2 g}\right) Z\left(\frac{2 \pi r_{o}}{\pi r_{0}^{2}}\right)$

and

$$
\tau_{\mathrm{E}}=\frac{\Delta P_{\mathrm{E}}}{Z}\left(\frac{r_{o}}{2}\right)
$$

where

$\Delta P_{E}=$ pressure drop due to profile drag, lb./sq.ft.

$\tau_{E}=$ shear due to profile drag, lb./ sq.ft.

$\rho_{G}=$ gas density, lb./ cu.ft.

$\mu_{a v g}=$ average velocity of gas stream based on superficial area, ft./sec.

Equation (33) is in a form which would permit one to predict profiledrag loss if the terms on the right- hand side are known. By adding profile drag to the skin friction the total pressure drop is obtained.

$$
\Delta P_{\mathrm{W}}=\Delta P_{\mathrm{E}}+\Delta P_{\mathrm{D}}
$$

where

$\Delta P_{w}=$ total pressure drop (wet), lb./sq.ft.

$\Delta P_{D}=$ pressure drop for dry tube, lb./sq.ft. 


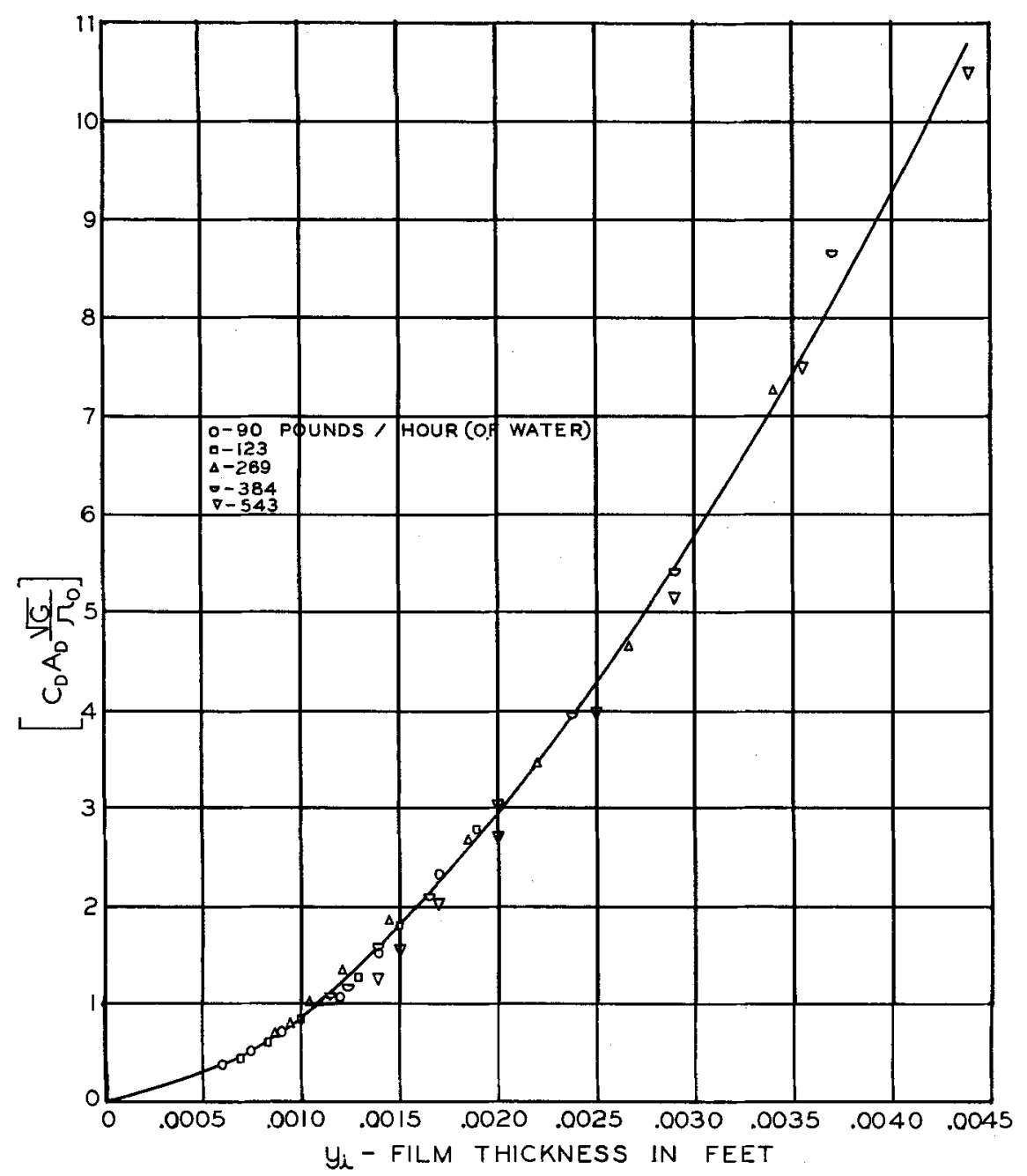

Fig. 6. RELATIONSHip BETWEen $C_{D} A_{D} \frac{\sqrt{G}}{r_{o}}$ AND $y_{i}$.
To predict $\Delta P_{B}$ one must know $C_{D}$ and $A_{D}$ under given conditions of flow. No analytical method is available for predicting these quantities, but an empirical correlation valid for the air-water system may be developed by use of the experimental data. The method of correlation is based on the assumption that $A_{D}$ is dependent mainly on the amount of water present in the tube and to some extent upon the velocity of the air.

Since $C_{b}$ will vary with the wave geometry and the gas velocity, it is combined with $A_{D}$ in this correlation as the term $\left(C_{D} A_{D}\right)$, which may be computed directly from experimental pressure-drop data. It was found from experimental data that $\left(C_{D} A_{D}\right)$ is proportional to $u_{A}{ }^{-\frac{1}{2}}$ and also dependent upon $y_{i}$. This relationship is graphically shown in Figure 6, a plot of $C_{D} A_{D} \frac{V G}{r_{o}}$ vs. $y_{i}$. By use of Figure 6 in conjunction with Equation (36) below, the pressure drop due to profile drag can be determined if air rate and density, tube diameter, and water-film thickness are known.

$$
\begin{gathered}
\Delta P_{\mathrm{E}}=\left(\frac{G}{r_{o}}\right)^{3 / 2}\left(\frac{\rho_{\mathrm{G}}}{(60 \pi)^{2} 2 g}\right) \\
\left(C_{\mathrm{D}} A_{\mathrm{D}} \frac{\sqrt{G}}{r_{o}}\right) \frac{2 Z}{r_{o}}
\end{gathered}
$$

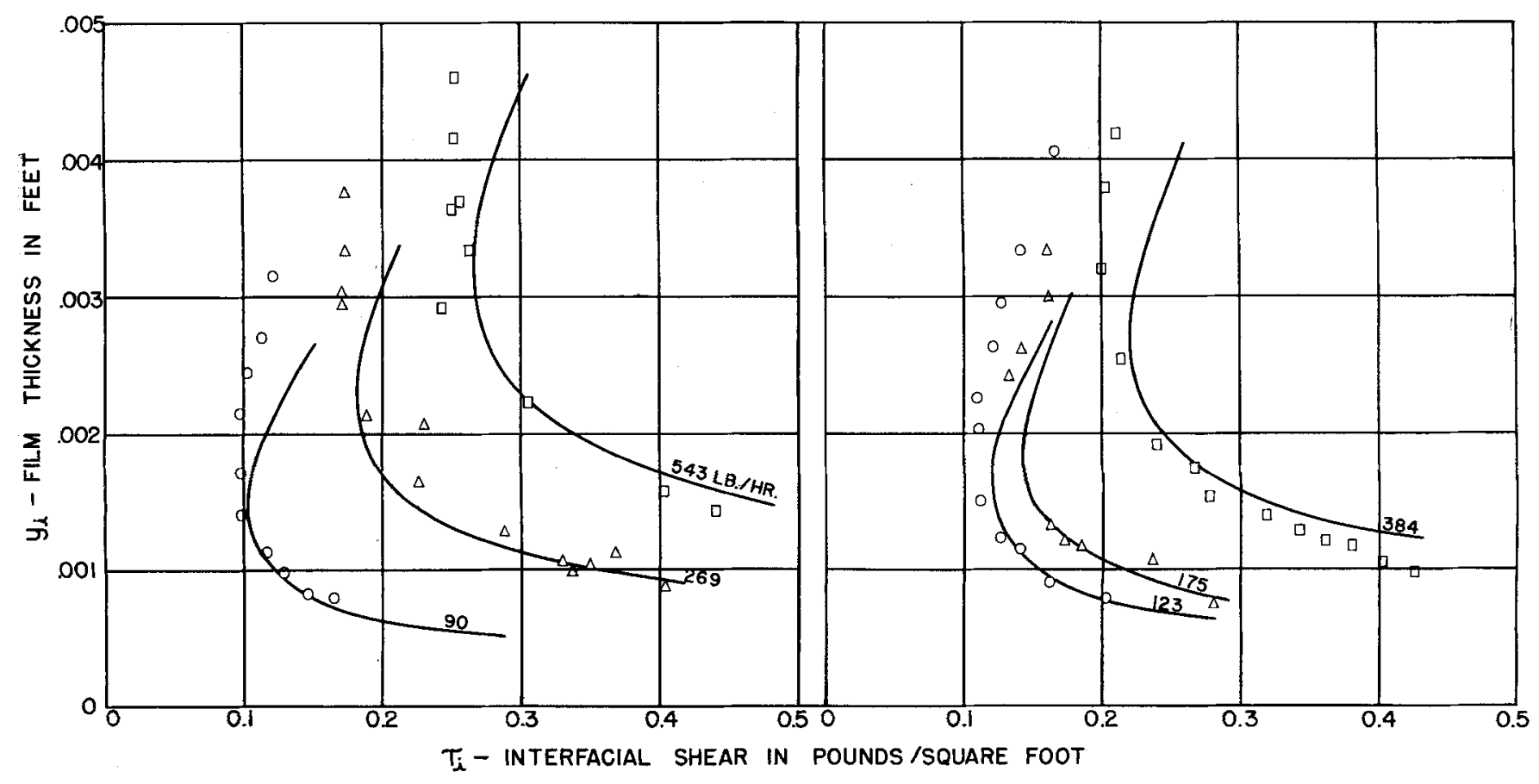

Fig. 7. Predicted Water-Film Thickness vs. Interfaclal Shear for Several Water Rates IN a 1.05-IN. I.D. Smooth Tube Compared with Experimental Points. 
To summarize the method of computing pressure drop for annular flow if $y_{i}, G, \rho_{G}, \mu_{G}$, and $r_{o}$ are known, the following procedure is given

1. Determine $\Delta P_{D}$ by the friction-factor method for the flow rate of $G$ through a $d r y$ tube of radius $r_{0}$.

2. Determine $\Delta P_{E}$ by use of Equation (36) and Figure 6.

3. Add $\Delta P_{E}$ and $\Delta P_{D}$ to get $\Delta P_{w}$, the pressure drop for annular flow.

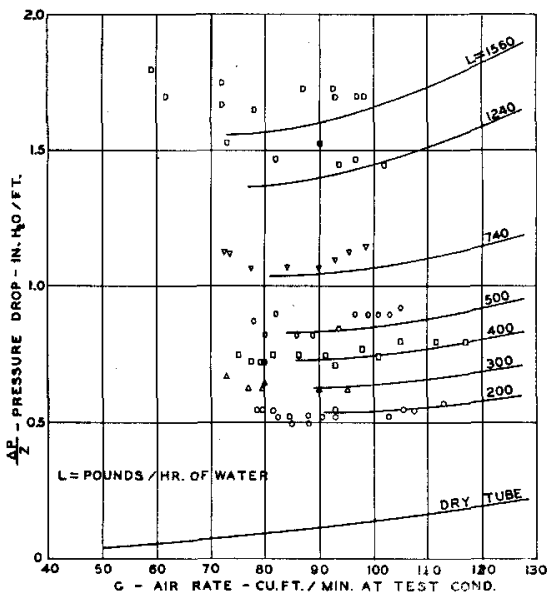

Fig. 8. COMPARISON OF EXPERIMENTAL With PRedicted Relationships Between Pressure Drop, Air Rate, AND WATER RATE FOR ANNULAR FLOW.

\section{COMPARISON OF PREDICTED WITH EXPERIMENTAL WATER- FILM THICKNESS}

The mathematical definition of vertical liquid-film flow was employed to predict water-film thickness as a function of interfacial shear and water-flow rate. The predictions for $90,123,175,269,384$, and $543 \mathrm{lb}$. of water/hr. in a 1 -in. I.D. tube are compared in Figure 7 with experimentally determined values. In the region of annular flow, that is, for film thicknesses smaller than those at the point of minimum shear, there is close agreement between the observed and predicted values.

\section{COMPARISON OF PREDICTED WITH EXPERIMENTAL PRESSURE DROP}

Values of pressure drop as a function of air rate for the flow of $200,300,400,500,740,1,240$, and $1,560 \mathrm{lb}$. of water/hr. in a 2 -in. I.D. tube were predicted by means of the method described. The predicted values are shown along with experimentally observed points on Figure 8, a plot of pressure drop vs. air rate with water rate as the parameter. The solid lines representing predicted values are discontinued at air rates lower than the minimum for annular flow.

\section{CONCLUSION}

The agreement between prediction and observation for both film thickness and pressure drop is sufficient to establish the validity of the methods of prediction. The deviation of both observed pressure drop and film thickness from the predicted values at high water rates is probably due to the occurrence of wave entrainment.

\section{ACKNOWLEDGMENT}

The authors gratefully acknowledge the permission of Dr. B. A. Radford to use his data on the holdup of liquid in two-phase flow.

\section{NOTATION}

$A=$ area, sq.ft.

$A_{o}=$ projected area for drag, sq.ft. /sq.ft.

$B=$ width of film, ft.

$C_{D}=$ drag coefficient

$C=$ a constant

$D=$ diameter, $\mathrm{ft}$.

$F=$ drag force, $\mathrm{lb}$.

$f=$ friction factor

$G=$ mass velocity, lb./ (hr.) (sq.ft.)

$g=$ acceleration of gravity

$k=l / y$

$l=$ Prandtl mixing length, ft.

$L=$ volume rate of flow

$N=$ a constant

$\Delta P=$ pressure drop $1 \mathrm{lb} . / \mathrm{sq} . \mathrm{ft}$.

$\Delta P_{E^{\prime}}=$ pressure drop due to drag, lb./sq.ft.

$\Delta P_{S}=$ stagnation pressure drop, lb. /sq.ft.

$R e=$ Reynolds number

$r=$ radius, $\mathrm{ft}$.

$u=$ velocity in axial direction at point $y$

$y=$ distance from solid boundary, ft.

$y_{i}=$ film thickness, ft.

$Z=$ height of film, ft.

\section{Subscripts}

$A=$ annular flow

$C=$ critical or transition of laminar to turbulent flow

$D=$ dry tube

$E=$ in profile drag

$G=$ gas phase

$i=$ gas-liquid interface

$L=$ liquid phase

lam = laminar flow

$o=$ tube wall $s=$ single-phase flow

$t=$ turbulent flow

$w=$ wet tube

Greek letters

$\delta=$ thickness of turbulent layer

$\mu=$ absolute viscosity

$p=$ density

$\tau=$ shear

\section{LITERATURE CITED}

1. Bakhmeteff, B. A., "The Mechanics of Turbulent Flow," Princeton University Press (1941).

2. Bergelin, O., Chem. Eng., 56, 104 (1949).

3. Boelter, C. M. R., and R. H. Kepner, Ind. Eng. Chem., 31, 426 (1939).

4. Cromer and Huntington, Trans. Am. Inst. Mining Met. Engrs., 136, 79 (1940).

5. Dukler, A. E., Ph.D. Thesis, Univ. Delaware (1951)

6. Fallah, R, T. G. Hunter, and A. W. Nash, J. Soc. Chem. Ind., 53, 367, T-379T (1934).

7. Friedman, S. J., and C. O. Miller, Ind. Eng. Chem., 33, 885 (1941).

8. Gazley, C., Ph.D. Thesis, Univ. Delaware (1948).

9. Gosline, J. E., Trans. Am. Inst. Mining Met. Engrs., 118, 56 (1936).

10. Jesser, B. W., and J. C. Elgin, Trans. Am. Inst. Chem. Engrs., 39, 277 (1943)

11. Keulegan, G. H., Natl. Bur. Standards Sci. Technol. Paper 2040, 43 (November, 1949).

12. Kinney and Abramson, Natl. Advisory Comm. Aeronaut. Tech. Mem. E51C13 (May, 1951).

13. Laufer, J., Natl. Advisory Comm. Aeronaut. Tech. Note 2123 (1950).

14. Martinelli, R. C., J. A. Putnam, and R. W. Lockhart, Trans. Am. Inst. Chem. Engrs., 42, 681 (1946).

15. Martinelli, R. C., L. M. K. Boelter, T. H. M. Taylor, E. G. Thomsen, and E. H. Morrin, Trans. Am. Soc. Mech. Engrs., 66, 139 (1944).

16. O'Brien, M. P., and J. E. Gosline, Ind. Eng. Chem., 27, 1436 (1935).

17. Prandtl, L., and O. G. Tietjens, "Applied Hydro- and Aerodynamies," McGraw-Hill Book Company, Inc., New York (1934).

18. Radford, B. A., M.S. Thesis, University of Alberta (1949).

19. Schlichting, H., Natl. Advisory Comm. Aeronaut., Tech. Mem. 823 (1937).

20. Stanton, T., Proc. Roy. Soc. (London), A 137, 283 (1932).

21. Tillman, W., Natl. Advisory Comm. Aeronaut. Tech. Mem. 1299 (1944).

22. Uren, L. C., P. P. Gregory, R. A. Hancock, and G. V. Feskov, Trans. Am. Inst. Mining Met. Engrs., 86, 209 (1930).

(Presented at A.I.Ch.E. Toronto meeting) 